\title{
Coronary Risk Factors and Oxidative Stress in Women with PCOS
}

\author{
Doddappa M Bannigida ${ }^{1}$, Shivananda B Nayak ${ }^{2}$
}

\begin{abstract}
Introduction: Polycystic ovary syndrome (PCOS) affects 5-10\% of women in reproductive age. It is associated with metabolic syndromes and non-metabolic disorders. Women with PCOS have dyslipidemia and oxidative stress representated by elevated serum Malonaldehyde (MDA) levels. These features are risk factors for development of atherogenesis and cardiovascular disease (CVD).

Materials and methods: A case-control study was conducted at the Department of Biochemistry, Koppal Institute of Medical Sciences, Koppal, India. It included 100 diagnosed PCOS patients (50- obese and 50 non-obese) and 100 controls (50-obese and 50 non-obese) in the age group of 18-40 years. The mean \pm SD were compared using student' $t$ ' - Test from the SPSS Statistics Data Editor version 21. Kruskal-Wallis test was employed for $p$ values. The $p$ value of $<0.05$ is considered statistically significant.

Results: Serum levels of total cholesterol, serum triglyceride, LDL, VLDL were higher in women with PCOS irrespective of BMI with $p$ value of $<0.001$.There were low levels of serum HDL in women with PCOS irrespective of BMI with $p$ value of $<0.001$. Serum MDA levels were higher in women with PCOS irrespective of BMI with $p$ value of $<0.001$.

Conclusion: Dyslipidemia and increased OS indicated by elevated levels of MDA are seen in women with PCOS irrespective of BMI. Hence, PCOS women should be evaluated for lipid profile and oxidative stress and should be treated with antioxidant supplementation, which can be beneficial in preventing coronary vascular diseases.

Keywords: BMI, CVD, Dyslipidemia, MDA.

Indian Journal of Medical Biochemistry (2019): 10.5005/jp-journals-10054-0101
\end{abstract}

\section{INTRODUCTION}

Polycystic ovary syndrome (PCOS) is the most common endocrine disease in women of reproductive age group with a wide spectrum of clinical manifestations. ${ }^{1-4}$ Women with PCOS have dyslipidemia because they have elevated androgen levels and are frequently obese along with hyperinsulinemia and insulin resistance. More than androgen levels of insulin levels correlate best with lipid abnormalities. Treatment of hyperandrogen levels does not alter lipid profiles in PCOS. ${ }^{5}$ Similar features are also seem to be associated with atherogenesis and cardiovascular disease (CVD). Increased plasma insulin levels induce more very low-density lipoprotein (VLDL) synthesis, causing hypertriglyceridemia. As the lipid and apolipoproteins are eliminated from the VLDL particle intermediate-density and low-density lipoproteins are formed, both of which are atherogenic. $^{5}$

Oxidative stress (OS) is associated with CVD as well as obese women with PCOS. Oxidative stress effects female reproductive system leading to infertility, ${ }^{6}$ thus takes part in the pathophysiology of PCOS. However, the functional cause of OS in PCOS is not clear. Studies suggest that the presence of IR and hyperglycemia in women with PCOS are main factors to cause OS. Oxidative stress defined as a disharmony between the production of ROS and antioxidant mechanism, which is an important component in PCOS women. 7

Polyunsaturated fatty acids (PUFA) present on cell membranes is attacked by oxidizing radicals, causing lipid peroxidation and malondialdehyde (MDA) produced is one of the stable end products of lipid peroxidation that can serve as a good biomarker. ${ }^{8}$

Alterations in the lifestyle such as weight loss and reduction of weight in women with PCOS stay the main component of treatment that improves BMI, menstrual disturbances, infertility, hyperandrogenism, and IR. ${ }^{9-11}$
${ }^{1}$ Tutor, ${ }^{2}$ Professor

${ }^{1}$ Department of Biochemistry, Koppal Institute of Medical Sciences, Koppal and Research scholar, Department of Research, Saveetha University, Thandalam, Chennai, TamilNadu, India

${ }^{2}$ Department of Preclinical Sciences, The University of the West Indies, Faculty of Medical Sciences, Trinidad and Department of Biochemistry, Subbaiah Institute of Medical Sciences, Shimoga, Karnataka, India

Corresponding Author: Shivananda B Nayak, Professor, Department of Preclinical Sciences, The University of the West Indies, Faculty of Medical Sciences, Trinidad and Department of Biochemistry, Subbaiah Institute of Medical Sciences, Shimoga, Karnataka, India, e-mail: shiv25@gmail.com

How to cite this article: Bannigida DM, Nayak SB. Coronary Risk Factors and Oxidative Stress in Women with PCOS. Indian J Med Biochem 2019;23(2):267-269.

Source of support: Nil

Conflict of interest: None

\section{Materials and Methods}

We conducted a case-control study at the Department of Biochemistry, Koppal Institute of Medical Sciences, Koppal, India from July 2015 to March 2018. It included 100 diagnosed PCOS patients (50 obese and 50 nonobese) and 100 controls (50 obese and 50 nonobese) in the age group of 18-40 years. Women suffering from any known diseases, any infections, and inflammatory conditions were excluded from the study. Fasting blood sample of $5.0 \mathrm{~mL}$ was obtained from cases and controls. Fasting lipid profile [total cholesterol, high-density lipoprotein cholesterol (HDL) and triglycerides] was done using enzymatic kits with biochemistry autoanalyzer (ERBA XL640). Low-density lipoprotein cholesterol (LDL) was calculated using the Friedwald formula. Serum malonaldehyde (MDA) was determined by Thiobarbituric acid reactive substances (TBARS). Ethical clearance

() The Author(s). 2019 Open Access This article is distributed under the terms of the Creative Commons Attribution 4.0 International License (https://creativecommons. org/licenses/by-nc/4.0/), which permits unrestricted use, distribution, and non-commercial reproduction in any medium, provided you give appropriate credit to the original author(s) and the source, provide a link to the Creative Commons license, and indicate if changes were made. The Creative Commons Public Domain Dedication waiver (http://creativecommons.org/publicdomain/zero/1.0/) applies to the data made available in this article, unless otherwise stated. 
was taken from the Ethics Committee of the college. Patients were informed about the research being conducted and asked to give their oral consent of participating in the study. Physical examination included height and weight of all individuals. Body mass index (BMI) was calculated as $\mathrm{kg} / \mathrm{m}^{2}$. Diagnosis of PCOS was done according to the Rotterdam ESHRE revised consensus 2003. Subjects included in the study were never on any hormonal contraceptives, aspirin, statins, vitamin supplements or any other significant drug therapy.

\section{Statistical Analysis}

The mean \pm SD were compared using student ' $\mathrm{t}$ '-test from the Statistical Package for Social Sciences (SPSS) statistics data editor version 21 . The $p$ value of $<0.05$ is considered statistically significant and $<0.001$ as highly significant.

\section{ResUlTs}

Total of 100 women diagnosed as having PCOS (50 obese and 50 non-obese) were compared with 100 controls without PCOS ( 50 Obese and 50 nonobese).

Serum levels of total cholesterol were higher in obese women with PCOS $275.08 \pm 20.2$ and nonobese women with PCOS $163.64 \pm$ 30.1 compared to their controls $192.96 \pm 13.2$ and $149.34 \pm 15$, respectively with $p$ value of $<0.001$ (Table 1 ).

Serum levels of serum triglyceride levels were higher in obese women with PCOS $167.03 \pm 36.74$ and nonobese women with PCOS $131.12 \pm 36.71$ compared to their controls respectively $130.70 \pm 32.8$ and $92.74 \pm 30.0$ with a $p$ value of $<0.001$ (Table 1 ).

There were low levels of serum HDL in obese women with PCOS $20.70 \pm 5.4$ and nonobese women with PCOS $28.80 \pm 8.45$ compared to their controls respectively $31.92 \pm 7.5$ and $37.02 \pm 7.5$, with $p$ value of $<0.001$ (Table 1).

Serum levels of serum LDL were higher in obese women with PCOS $141.51 \pm 26.8$ and nonobese women with PCOS $127.97 \pm 24.96$ compared to their controls respectively $119.46 \pm 32.99$ and $57.37 \pm$ 38.20 with $p$ value of $<0.001$ (Table 1).

There were higher levels of serum VLDL in obese women with PCOS $33.25 \pm 7.27$ and nonobese women with PCOS $26.23 \pm 7.39$ compared to their controls respectively $26.05 \pm 6.51$ and $26.23 \pm$ 6.02 with $p$ value of $<0.001$ (Table 1).

Serum MDA levels were higher in obese women with PCOS $7.14 \pm 0.54$ and nonobese women with PCOS $5.54 \pm 0.32$ compared to their controls $3.96 \pm 0.42$ and $1.91 \pm 0.40$, respectively with $p$ value of $<0.001$ (Table 1).

\section{Discussion}

Dyslipidemia was observed in both obese and nonobese PCOS cases compared to their controls. Similar observations were made by other studies. ${ }^{12-18}$

PCOS patients exhibit oxidative stress due to hyperglycemia, IR and chronic inflammation, that leads to overproduction of ROS. Hyperglycemia produces tumor necrosis factor a (TNF a) from multinuclear cells (MNC) thus playing a role to inflammation. Some studies conducted on healthy women with hyperglycemia have higher levels of ROS due to androgenic stimulation of leukocytes, p47phox gene expression, and formation of MDA. Nonobese women with PCOS also exhibit diet-induced OS with hyperandrogenism being the progenitor. OS and chronic inflammation express a vicious cycle. ${ }^{19}$

Serum level of MDA which is the end product of lipid peroxidation was significantly elevated in both obese and nonobese PCOS. Similar observations were made by various other studies. ${ }^{14-23}$

As levels of MDA correlate with the extent of lipid peroxidation, it can serve as a common biomarker to assess the oxidant status. Free radicals in the body stay for a short duration before achieving stability by colliding with another molecule to either receive or donate an electron, eventually generating free radical (ROS). These ROS target proteins, carbohydrates, nucleic acids and polyunsaturated fatty acids (PUFA), present in the cell membrane forming various end products. This process is opposed by antioxidant enzymes thus maintaining the balance of the cell. The loss of harmony between oxidants and antioxidants leads to oxidative stress. ${ }^{19}$

\section{ConCLusion}

Dyslipidemia and increased levels of serum MDA represent OS seen in women with PCOS irrespective of BMI. Hence, PCOS women should be evaluated for lipid profile, oxidative stress and should be treated with antioxidant supplementation, which can be beneficial in preventing coronary vascular diseases.

This study can deepen our knowledge about the key role of dietary modifications and drug treatments of metabolic disorders in improving the cardiovascular risk in PCOS woman. Detection of inflammatory markers can reduce the overall morbidity and enhances the prognosis of PCOS.

\section{Clinical significance}

Treating physicians should be aware of coronary heart disease in women with PCOS.

Table: 1 Characteristics in women with PCOS and controls

\begin{tabular}{|c|c|c|c|c|c|c|}
\hline \multirow[b]{2}{*}{ Characteristics } & \multicolumn{3}{|c|}{ Non-obese } & \multicolumn{2}{|c|}{ Obese } & \multirow[b]{2}{*}{$p$ value } \\
\hline & Cases & Control & $p$ value & Cases & Control & \\
\hline Age (Years) & $23.0 \pm 2.99$ & $25.2 \pm 3.44$ & & $25.4 \pm 4.12$ & $24.5 \pm 2.11$ & - \\
\hline BMI $\left(\mathrm{kg} / \mathrm{m}^{2}\right)$ & $25.2 \pm 4.86$ & $21.6 \pm 2.53$ & & $35.2 \pm 4.39$ & $32.7 \pm 3.05$ & - \\
\hline Cholesterol (mg/dL) & $\begin{array}{l}185.00 \\
(156,193)\end{array}$ & $\begin{array}{l}108.50 \\
(94.75,128.00)\end{array}$ & $<0.001$ & $\begin{array}{l}196.00 \\
(184.00,213.00)\end{array}$ & $\begin{array}{l}184.00 \\
(151.00,198.50)\end{array}$ & $<0.001$ \\
\hline TGL (mg/dL) & $131.12 \pm 36.71$ & $92.74 \pm 30.0$ & $<0.001$ & $167.03 \pm 36.74$ & $130.70 \pm 32.8$ & $<0.001$ \\
\hline $\mathrm{HDL}(\mathrm{mg} / \mathrm{dL})$ & $28.80 \pm 8.45$ & $37.02 \pm 7.5$ & $<0.001$ & $20.70 \pm 5.4$ & $31.92 \pm 7.5$ & $<0.001$ \\
\hline LDL (mg/dL) & $127.97 \pm 24.96$ & $57.37 \pm 38.20$ & $<0.001$ & $141.51 \pm 26.8$ & $119.46 \pm 32.99$ & $<0.001$ \\
\hline VLDL (mg/dL) & $26.23 \pm 6.02$ & $18.56 \pm 7.39$ & $<0.001$ & $33.25 \pm 7.27$ & $26.05 \pm 6.51$ & $<0.001$ \\
\hline $\mathrm{MDA}(\mathrm{nmol} / \mathrm{mL})$ & $5.54 \pm 0.32$ & $1.91 \pm 0.40$ & $<0.001$ & $7.14 \pm 0.54$ & $3.96 \pm 0.42$ & $<0.001$ \\
\hline
\end{tabular}




\section{ACKNOWLedgments}

Authors wish to express their indebtedness to the Koppal Institute of Medical Sciences, Koppal and their laboratory staff for their technical support.

\section{References}

1. Tehrani FR, Rashidi H, Khomami MB, Tohidi M, Azizi F. The prevalence of metabolic disorders in various phenotypes of polycystic ovary syndrome: a community based study in Southwest of Iran. Reproductive biology and endocrinology. 2014;12(89):1-6.

2. Kitzinger C, Willmott J.'The thief of womanhood': women's experience of polycystic ovarian syndrome. Social science and medicine. 2002; 54(3):349-361.

3. Castro AV, Kolka CM, Kim SP, Bergman RN. Obesity, insulin resistance and comorbidities? Mechanisms of association. Arquivosbrasileiros de endocrinologia e metabologia. 2014;58(6):600-609.

4. Velez LM \&Motta AB. Association between polycystic ovary syndrome and metabolic syndrome. Current medicinal chemistry. 2014; 21(35): 3999-4012.

5. DeFronzo RA, Ferrannini E. Insulin resistance. A multifaceted syndrome responsible for NIDDM, obesity, hypertension, dyslipidemia, and atherosclerotic cardiovascular disease. Diabetes Care. 1991; 14(3):173194.

6. Agarwal A, Gupta S, Sharma RK. Role of oxidative stress in female reproduction. Reprod Biol Endo Crinol. 2005;3(28):1-21.

7. Sabuncu T, Vural H, Harma M, Harma M. Oxidative stress in polycystic ovary syndrome and its contribution to the risk of cardiovascular disease. Clin Biochem. 2001;34:407-413.

8. Mahfouz R, Sharma R, Sharma D, Sabanegh E, Agarwal A. Diagnostic value of the total antioxidant capacity (TAC) in human seminal plasma. Fertil Steril 2009; 91(3):805-811.

9. Moran LJ, Hutchison SK, Norman RJ, Teede HJ. Lifestyle changes in women with polycystic ovary syndrome. The Cochrane database of systematic reviews. 2011;2:7-10.

10. Moran LJ, Hutchison SK, Norman RJ, Teede HJ. Lifestyle changes in women with polycystic ovary syndrome 2011;7:58-65.

11. Huber-Buchholz MM \& Norman RJ. Restoration of Reproductive Potential by Lifestyle Modification in Obese Polycystic Ovary Syndrome: Role of Insulin Sensitivity and Luteinizing Hormone. The Journal of Clinical Endocrinology \& Metabolism. 2013;84(4): 1470-1474.
12. Zuo T, Zhu M, Xu W. Roles of Oxidative Stress in Polycystic Ovary Syndrome and Cancers. Oxidative Medicine and Cellular Longevity. 2016;1:1-15.

13. Desai V, Prasad NR, Manohar SM, Sachan A, Lakshmi Narasimha SRP, Bitla ARR. Oxidative Stress in Non-Obese Women with Polycystic Ovarian Syndrome. Journal of Clinical and Diagnostic Research. 2014; 8(7): 1-3.

14. Karabulut AB, Cakmak M, Kiran RT, Sahin I. Oxidative Stress Status, Metabolic Profile and Cardiovascular Risk Factors in Patients with Polycystic Ovary Syndrome. Medicine Science. 2012;1(1):27-34.

15. Valkenburg TO, Regine PM, Theunissen S, Huberdina PM, Smedts, Dallinga-Thie GM et al. A More Atherogenic Serum Lipoprotein Profile Is Present in Women with Polycystic Ovary syndrome: A Case-Control Study. J Clin Endocrinol Metab. February 2008; 93(2):470-476.

16. Macut D, Panidis D, Glisic B, Spanos N, Petakov M, Bjekic J, Bozic I, Damjanovic $S$. Relation of proatherogenic lipid profile and insulin resistance in PCOS. Endocrine Abstracts. 2008;16:646-652.

17. Kalra A, Nair S, Rai L. Association of obesity and insulin resistance with dyslipidemia in Indian women with polycystic ovarian syndrome. Indian journal of medical sciences 2006;60(11):447-453.

18. Unni NC, Sumithra, Lakshmi RL, Menon NL, Subhakumari KN, Sheejamol VS. Evaluation of Oxidative Stress and hsCRP in Polycystic Ovarian Syndrome in a Tertiary Care Hospital. Ind J Clin Biochem. 2015;30(2):161-166.

19. Deba1 Z, Jambale TA, Gangadhara Swamy PM, Murthy DSJ. Study of levels of malondialdehyde, super oxide dismutase and hsCrp in serum of non-obese patients with polycystic ovarian syndrome International Journal of Clinical Biochemistry and Research. 2017; 4(2):191-194

20. Mandal B, Bandyopadhyay R, Chakrabarti S, Bandyopadhyay TK. Assessment of Oxidative stress in Patients of Diabetes Mellitus with and without Complications. Journal of Indian Academy of Clinical Medicine. 2010;11(1):20-25.

21. Zhang D, Luo WY, Liao H, Wang CF, Sun Y. The effects of oxidative Stress to PCOS. Sichuan Da Xue Xue Bao Yi Xue Ban 2008; 39(3):421-423.

22. Palacio JR, Iborra A, Ulcova-Gallova Z, Badia R, Martinez P. The presence of antibodies to oxidative modified proteins in serum from polycystic ovary syndrome patients. Clin Exp Immunol 2006; 144(2): 217-222.

23. Fenkci V, Fenkci S, Yilmazer M, Serteser M. Decreased total antioxidant status and increased oxidative stress in women with polycystic ovary syndrome may contribute to the risk of cardiovascular disease. Fertil Steril 2003;80(1):123-127. 